\title{
CÁLCULO DE PESSOAL DE ENFERMAGEM: COMPETÊNCIA DA ENFERMEIRA
}

\author{
Maria Coeli Campedelli* \\ Clarinda Takito** \\ Tânia Regina Sancinetti*** \\ Maria Antonieta Benko****
}

\begin{abstract}
RESUMO - As autoras analisam a problemática enfrentada pela Direção de Enfermagem de um hospital frente ao número de pessoal na prestação de assistência. Enfatizam que fórmulas para o cálculo de pessoal devem ser utilizadas considerando: qualidade de assistência pretendida, assistência que os pacientes necessitam e características da Instituição. Cada hospital poderá utilizar as fórmulas pré-estabelecidas, numa fase inicial, mas de acordo com o percentual de cuidados que os pacientes requeiram e mudanças que interfiram na assistência, os cálculos devem ser refeitos. Este trabalho pretende, de forma modesta, utilizando exemplos reais, fornecer subsídios para que os enfermeiros possam discutir junto à administração ou outros serviços que fazem interferência na sua área, o cálculo de pessoal de enfermagem, que é de sua competência.
\end{abstract}

\begin{abstract}
The authors analyze the problem faced by administration of the Nursing sector of a hospital, in terms of number of personnel. They emphasize that, if estimating formulae are used, these points must be considered: quality of assistance intended, assistance needed by the patients and characteristics of the Institution. Each hospital may use pre-establised formulae, in a first phase, but, depending on the percentage of care requerid by the patients and the general changes that may interfere with the assistance, this calculation must be remade. The purpose of this work, in a modest way, is to provide data, making use of real examples, sothat nurses are able to discuss with the administration or other sectors that may interfere with the Nursing area-about the estimating of the Nursing personel, which is their attribution.
\end{abstract}

\section{INTRODUÇÃO}

No desempenho de seu papel profissional, a enfermeira tem buscado continuamente assistir à saúde do homem de forma cada vez mais cientifica e independente. Esta busca ocorre tanto em situaçōes novas, como no reestudo daquelas que a prática tem como consagradas. A dotação de recursos humanos para a prestação da assistência de enfermagem é uma das suas atribuiçōes já estabelecidas mas, que constitui muitas vezes, um problema para a enfermeira que responde pela gerência da assistência nos hospitais onde se discute $o$ assunto. Inúmeras são as dificuldades encontradas para o desempenho desta coordenação.

$O$ pessoal de enfermagem representa um percentual importante em relação ao número total dos servidores no hospital. Segundo FELDMANN (s.d.) e DUTRA (1983), este índice é igual a $60 \%$, o que representa um custo elevado e envolve um processo contínuo de admissão seleção, treinamento, aperfeiçoamento e avaliação. Cria, por motivos econômicos, políticos e outros, a interferência de profissionais de outras áreas na determinação da quantidade e qualidade do pessoal. Outra dificuldade que pode ocorrer, é a ausência ou indefinição de programas e objetivos da Instituição ou do conflito desta com os objetivos do serviço de enfermagem. É necessário identificar a causa da interferência, que não raro leva à ingerência, para demonstrar os riscos operacionais de um serviço programado inadequadamente e o comprometimento legal que a Instituição assume diante dos inúmeros perigos e falhas, que ocorrem tanto com o paciente quanto com o pessoal que o assiste. Portanto, a dotação de pessoal, além de ser da competência da enfermeira, representa uma preocupação constante para a enfermagem visto que está diretamente ligada ao produto final de seu trabalho

\footnotetext{
* Professora Assistente da Escola de Enfermagem da U.S.P. e Diretora do Departamento de Assistência de Enfermagem do Hospital Universitário da U.S.P.

** Professora Assistente da Escola de Enfermagem da U.S.P. e Supervisora de Ensino e Pesquisa do Hospital Universitário da U.S.P.

*** Diretor de Serviço do Pronto Atendimento do Departamento de Assistência de Enfermagem do Hospital Universitário da U.S.P; **** Diretor Técnica de Divisão do Departamento de Assistência de Enfermagem do Hospital Universitário da U.S.P.
} 
- a qualidade de assistência prestada e a segurança do paciente.

A preocupação com a qualidade da assistência de enfermagem a ser prestada é enfatizada por diversos autores, entre os quais ALCALA (1982), DUTRA (1983), OGUISSO (1970) e FELDMANN (s.d.). Este assunto requer da enfermeira conhecimento específico, habilidade na sua condução e segurança nas justificativas de suas propostas. Estas propostas são encontradas em estudos realizados e na análise de situações vivenciadas.

O tempo necessário à prestação da assistência de enfermagem varia segundo autor e tipo de paciente. De acordo com OGUISSO (1970), a necessidade de hora/enfermagem é a seguinte: Pediatria 4,9h; Clínica Médica 2,2h; Clínica Cirúrgica 2,4h; U.T.I. 7,1h. FELDMANN (s.d.) estabelece para Pediatria 4,9h/ Clínica Médica 3,4h; Clínica Cirúrgica 3,5h; Obstetrícia 4,2h. ABBOUD (s.d.) estabelece os mesmos indices de FELDMANN (s.d.), mas acrescenta $12 \mathrm{~h}$ para a U.T.I.. ATALA (1978) também apresenta valores aproximados: Pediatria 3 e 4h; Clínica Médica 2,5 à 3h; Clínica Cirúrgica 3h; Obstetrícia 2h e U.T.I. 12,4h. Já em 1982, ALCALA realizou um estudo para o estabelecimento do quadro de pessoal da Superintendência Médico Hospitalar de Urgência do Munićpio de São Paulo, partindo do tipo de cuidado prestado a cada paciente, com base na Filosofia do Cuidado Progressivo ao $\mathrm{Pa}-$ ciente. Estabeleceu a hora/enfermagem para o paciente Adulto e Pediátrico: cuidados mínimos 2,5h; intermediário $4,5 \mathrm{~h}$; semi-intensivo $10 \mathrm{~h}$ e intensivo $18 \mathrm{~h}$ e para a Pediatria $4,5 \mathrm{~h} ; 5,5 \mathrm{~h}$; $10 \mathrm{~h}$ e $18 \mathrm{~h}$ respectivamente. Pode-se observar que a variação da hora/enfermagem entre os diversos autores é pequena, e muitas vezes não traduz a realidade do dia-a-dia, que pode oscilar muito de acordo com a gravidade dos pacientes, exceção feita por ALCALA (1982) que se preocupou exatamente com este aspecto.

Com relação ao percentual de distribuição por categoria funcional há também diversidade de opiniāo entre os diversos autores: ALCALA (1982) preconiza $15 \%$ enfermeiros, $45 \%$ auxiliares de enfermagem e $40 \%$ atendentes; FELDMANN (s.d.), $10 \%$ enfermeiros, $20 \%$ auxiliares de enfermagem e $60 \%$ atendentes. No material preparado pelo prof essor Miguel ABBOUD (s.d.) para o Curso de Administração Hospitalar da Escola de Administração de Empresas de São Paulo da Fundação Getúlio Vargas e Hospital das Clínicas da Faculdade de Medicina da Universidade de São Paulo, o preconizado para todas as unidades incluindo emergência é de 5\% enfermeiros, $10 \%$ técnicos de enfermagem, $25 \%$ auxiliares de enfermagem e $60 \%$ atendentes. Para as Unidades de Terapia Intensiva, este percentual tem a seguinte distribuição: $10 \%$ enfermeiros, $20 \%$ técnicos de enfermagem, $60 \%$ auxiliares de enfermagem e $10 \%$ atendentes.
Este percentual quando entra em discussão, do ponto de vista administrativo de custo, deve levar em conta a qualidade de assistência que se pretende prestar, pois esta é diretamente dependente da qualificação profissional e ocupacional dos funcionários.

O Hospital Universitário da Universidade de São Paulo conta atualmente com 1252 cargos de funcionários, dos quais 450 são destinados à enfermagem, representando $35,94 \%$ do pessoal, portanto com um percentual abaixo do proconizado. A distribuição funcional é a seguinte: 100 enfermeiros $(22,22 \%) 15$ técnicos de enfermagem $(3,33 \%), 101$ auxiliares de enfermagem $(22,44 \%), 213$ atendentes de enfermagem $(47,33 \%), 19$ escriturários $(4,22 \%)$ e 01 recreacionista $(0,2 \%)$.

Retirando-se do quadro do Hospital Universitário as duas categorias não tradicionalmente inclurdas no quadro funcional de enfermagem (19 escriturários e 01 recreacionista), teremos 430 funcionários distriburdos da forma seguinte: $23 \%$ enfermeiros, $3,5 \%$ técnicos de enfermagem, $23,5 \%$ auxiliares de enfermagem e $50 \%$ atendentes de enfermagem.

O Departamento de Assistência de Enfermagem do Hospital Universitário tem, estabelecido em sua filosofia, a adoção de uma sistemática de assistência de enfermagem para o desempenho de suas atividades junto ao paciente. A adoção de uma sistemática de assistência, com a prescrição diária do cuidado, está vinculada ao cumprimento de um dispositivo legal do exercício profissional da enfermeira, segundo a qualidade da assistência a ser prestada e deve ser considerada na elaboração do quadro de pessoal.

À medida que se procura diferenciar o cuidado de enfermagem necessário a cada paciente, varia o número de horas de enfermagem gasto e, como conseqüência, a quantidade e a qualidade de pessoal também será diferente. Esta variação sofre questionamentos e por isso é necessário estipular padrões, que em consonância com a filosofia do serviço, mobilizem o tipo de assistência que se pretende prestar. A vivência desta problemática deu origem a este trabalho, que tem como objetivo maior, estimar o número de funcionários de enfermagem necessários à prestação da assistência de enfermagem do Hospital Universitário de São Paulo, utilizando três índices diferentes. Para realizar este objetivo, foram adotados para cálculo dois índices propostos por diferentes autores: 1. ABBOUD (s.d.), 2. ALCALA (1982), sendo que o terceiro índice utilizado foi extrardo da realidade identificada no Hospital Universitário.

\section{METODOLOGIA}

O estudo foi realizado no Hospital Universitário da Universidade de São Paulo em duas clínicas de pacientes internados: Clínica Médica e Clínica Pe- 
diátria, localizadas respectivamente no $5^{\circ}$ e $4^{\circ}$ pavimentos.

A população de estudo foi constituída por todos os pacientes hospitalizados nas duas clínicas durante o período de um mês.

As duas clínicas que constituiram o campo de estudo têm gerência própria de pessoal, material e movimentação de pacientes.

Cada uma delas conta com duas unidades de internação, com posto de enfermagem, sala de serviço e outras dependências próprias de uma unidade de internação. $\mathrm{O}$ número de leitos em ambas as clínicas, no mês de estudo, foi de 35 leitos.

Os leitos, na Clínica Médica, estão distribuŕdos em quartos individuais e enfermarias de cinco leitos, estando uma delas equipadas para cuidados especiais a pacientes graves.

A Clínica Pediátrica é constiturda também por duas unidades de internação com enfermaria de cinco leitos, quartos individuais, uma enfermaria para 12 berços e uma unidade para cuidados especiais.

Os quartos individuais, nas duas clínicas, são geralmente ocupados por pacientes que requerem algum tipo de isolamento fisiológico ou emocional.

Para calcular o número de pessoal necessário foi utilizada sempre a mesma fórmula:

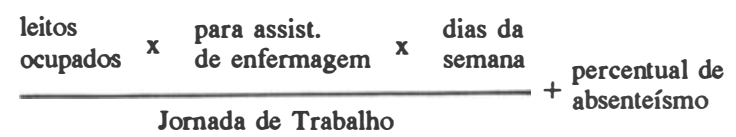

onde:

- leitos ocupados é o correspondente ao percentual de leitos ocupados, em relação ao total de leitos existentes, preconizado ou pelo autor ou encontrado no Hospital Universitário;

- horas assistência de enfermagem é igual ao número de hora/enfermagem dispendidos com o cuidado ao paciente nas 24 horas e varia por autor ou tipo de cuidado para adulto e pedatria;

- dias da semana, utilizam-se sete;

- jornada de trabalho, utiliza-se o número de horas semanais trabalhadas $(40 \mathrm{~h})$;

- percentual de absenteŕsmo corresponde à cobertura das ausências ocasionadas por licenças, faltas, férias e folgas. Também varia por autor ou encontrado no Hospital Universitário.

- Para registrar os dados necessários à determinação do tipo de cuidados que o paciente requer, foi elaborado um mapa com identificação da clínica, contendo trinta e uma caselas correspondentes ao número de dias da coleta, para serem preenchidas com o número de leitos ocupádos em função do tipo de cuidado. Estes pacientes foram classificados usando as definições estabelecidas por ALCALA (1982).

As enfermeiras que participaram do plano piloto foram as mesmas que realizaram a coleta de dados. 0 ensaio preliminar foi executado durante os 15 dias que antecederam à realização do estudo nas mesmas unidades de internação, permitindo avaliar o instrumento, preparar as enfermeiras na determinação do tipo de cuidados e mostrar a viabilidade do mesmo.

A coleta de dados foi realizada, durante a visita diária aos pacientes, às 8 horas, por três enfermeiras da Clínica Médica e duas enfermeiras da Clínica Pediátrica. Nesta visita que é de rotina no HU, a enfermeira faz uma entrevista, exame físico sumário, evolução e prescrição de enfermagem.

Durante a entrevista e exame físico, na fase de realização do estudo, eram identificadas as condições de movimentação e deambulação, tipo de isolamento, presença de sondas, drenos, cateteres, renoclises, curativos, lesões, orientação auto e halo psíquica e condições emocionais dos pacientes. Na Pediatria levou-se ainda em consideração o tipo de berço (isolete, berço aquecido ou berço comum). $\mathrm{Na}$ avaliação e prescrição de enfermagem era feita uma comparação com a evolução dos dias anteriores, e verificava-se o aparecimento e resolução de novos problemas, assim como o número de cuidados a serem prestados. Cada paciente foi visitado tantas vezes quantos os dias em que permaneceu hospitalizado neste mês de estudo.

Para calcular o percentual de absenteŕsmo em ambas as clínicas, utilizou-se a formula encontrada em MEZOMO (1981) onde:

Taxa de absent.

$$
=\frac{\text { hom } / \text { dia perd. } \times 100}{\text { hom/dias trab. }+ \text { hom/dias perdidos }}
$$

Considerou-se para homem/dia perdido: folgas, férias, faltas, licenças, vagas por demissõese para homem/dia trabalhado, os 31 dias trabalhados de cada funcionário no mês de maio.

O resultado foi, para a Pediatria de $33 \%$ de absenteŕsmo e para a Clínica Médica, de $37 \%$.

\section{RESULTADOS}

Para facilitar a compreensão dos resultados optou-se por apresentar os cálculos elaborados, utilizando-se os índices por autor, sendo: 1. ABBOUD (s.d.), 2. ALCALA (1982) e 3. encontrados no HU, para a Clínica Pediátrica e Clínica Médica, seguidos das tabelas com distribuição funcional.

\section{Clínica Pediátria:}

1. 35 leitos: $80 \%$ de ocupação: $20 \%$ de absenteísmo. $28 \times 4,9 \times 7$ 40

Pelos índices recomendados por ABBOUD (s.d.), obteve-se um total de 29 funcionários.

2. 35 leitos: $80 \%$ de ocupação; $30 \%$ de absentermos; e com $60 \%$ dos leitos ocupados para cui- 
dados mínimos, $30 \%$ para cuidados intermediários e $10 \%$ para cuidados intensivos.

a. Cuidados Mínimos =

$$
\frac{16,8 \times 4,5 \times 7}{40}+30 \%=17,20
$$

b. Cuidados Intermediários =

$$
\frac{8,4 \times 5,5 \times 7}{40}+30 \%=10,50
$$

c. Cuidados Semi-intensivos $=$

$$
\frac{2,8 \times 10 \times 7}{40}+30 \%=6,37
$$

Realizado o cálculo, de acordo com os índices estabelecidos por ALCALA (1982), obteve-se um total de 34,07 funcionários.

3. 35 leitos: $91,42 \%$ de ocupação e $33 \%$ de absenteísmo.

Utilizando a classificação de ALCALA (1982), mas com a distribuição de ocupação em $6,25 \%$ para cuidados mínimos; $15,62 \%$ para cuidados intermediários; $65,62 \%$ para cuidados semi-intensivos e $12,5 \%$ para cuidados intensivos, resultado dos dados colhidos no mês em estudo:

a. Cuidados Mínimos =

$$
\frac{2 \times 4,5 \times 7}{40}+33 \%=2,09
$$

b. Cuidados Intermediários $=$

$$
\frac{5 \times 5,5 \times 7}{40}+33 \%=6,40
$$

c. Cuidados Semi-intensivos $=$

$$
\frac{21 \times 10 \times 7}{40}+33 \%=48,87
$$

d. Cuidados Intensivos $=$

$$
\frac{4 \times 18 \times 7}{40}+33 \%=16,75
$$

Realizado o cálculo, de acordo com os índices encontrados no HU, obteve-se um total de 74,11 funcionários.

\section{Clínica Médica}

1. 35 leitos: $80 \%$ de ocupação e $20 \%$ de absente-

ísmo.

$\frac{28 \times 3,4 \times 7}{40}+20 \%=19,99$
Pelos índices recomendados por ABBOUD (s.d.), obteve-se um total de 20 funcionários.

2. 35 leitos: $80 \%$ de ocupação; $30 \%$ de absenteísmo e com $30 \%$ dos leitos ocupados para cuidados mínimos; $60 \%$ para cuidados intermediários e $30 \%$ para cuidados semi-intensivos.

a. Cuidados Mínimos =

$$
\frac{8,4 \times 2,5 \times 7}{40}+30 \%=4,76
$$

b. Cuidados Intermediários $=$

$$
\frac{16,8 \times 4,5 \times 7}{40}+30 \%=17,29
$$

c. Cuidados Semi-intensivos =

$$
\frac{2,8 \times 10,0 \times 7}{40}+30 \%=6,37
$$

Realizado o cálculo, de acordo com os índices estabelecidos por ALCALA (1982), obteve-se um total de 28,42 funcionários.

3. 35 leitos: $91,52 \%$ de ocupação e $37 \%$ de absenteísmo. utilizamos a classificação de ALCALA (1982) mas com a distribuição de ocupação em $53,12 \%$ de cuidados mínimos; $34,73 \%$ de cuidados intermediários; $9,37 \%$ de cuidados semi-intensivos e $3,12 \%$ de cuidados intensivos, que foi a nossa realização do mês em estudo.

a. Cuidados Mínimos =

$$
\frac{17 \times 2,5 \times 7}{40}+37 \%=10,18
$$

b. Cuidados Intermediários $=$

$$
\frac{11 \times 4,5 \times 7}{40}+37 \%=11,86
$$

c. Cuidados Semi-intensivos =

$$
\frac{3 \times 10 \times 7}{40}+37 \%=7,19
$$

d. Cuidados Intensivos $=$

$$
\frac{1 \times 18 \times 7}{40}+37 \%=4,31
$$

Realizado o cálculo, de acordo com os índices encontrados no HU, obteve-se um total de 33,54 funcionários. 
Tabela 1

Distribuiçăo do pessoal de enfermagem, por categoria funcional segundo ABBOUD, ALCALA e dados do Departamento de Assistência de Enfermagem do Hospital Universitário, para a Clínica Pediátrica, 1986.

\begin{tabular}{|c|c|c|c|c|c|c|}
\hline \multirow[b]{2}{*}{$\begin{array}{l}\text { Categoria } \\
\text { Funcional }\end{array}$} & \multicolumn{2}{|c|}{ ABBOUD } & \multicolumn{2}{|c|}{ ALCALA } & \multicolumn{2}{|c|}{$\begin{array}{l}\text { Departamento de } \\
\text { Assistência de } \\
\text { Enfermagem - HU }\end{array}$} \\
\hline & $\begin{array}{l}\text { Indice } \\
\text { preconizado } \\
\text { em } \%\end{array}$ & $\begin{array}{l}\text { Valor } \\
\text { Absoluto }\end{array}$ & $\begin{array}{l}\text { Indice } \\
\text { preconizado } \\
\text { em } \%\end{array}$ & $\begin{array}{c}\text { Valor } \\
\text { Absoluto }\end{array}$ & $\begin{array}{l}\text { Índice } \\
\text { preconizado } \\
\text { em } \%\end{array}$ & $\begin{array}{c}\text { Valor } \\
\text { Absoluto }\end{array}$ \\
\hline $\begin{array}{l}\text { Enfermeira } \\
\text { Técnico de Enfermagem } \\
\text { Auxiliar de Enfermagem } \\
\text { Atendente de Enfermagem }\end{array}$ & $\begin{array}{r}5 \\
10 \\
25 \\
60\end{array}$ & $\begin{array}{r}2 \\
3 \\
7 \\
17\end{array}$ & $\frac{15}{45}$ & $\begin{array}{l}5 \\
15 \\
14\end{array}$ & $\begin{array}{l}23 \\
3,5 \\
23,5 \\
50\end{array}$ & $\begin{array}{r}17 \\
3 \\
17 \\
37\end{array}$ \\
\hline TOT A L & 100,0 & 29 & 100,0 & 34 & 100,0 & 74 \\
\hline
\end{tabular}

Tabela 2

Distribuiçăo do pessoal de enfermagem, por categoria funcional segundo ABBOUD, ALCALA e dados do Departamento de Assistência de Enfermagem do Hospital Universitário, para a Clínica Médica, 1986.

\begin{tabular}{|c|c|c|c|c|c|c|}
\hline \multirow[b]{2}{*}{$\begin{array}{l}\text { Categoria } \\
\text { Funcional }\end{array}$} & \multicolumn{2}{|c|}{ ABBOUD } & \multicolumn{2}{|c|}{ ALCALA } & \multicolumn{2}{|c|}{$\begin{array}{l}\text { Departamento de } \\
\text { Assistência de } \\
\text { Enfermagem - HU }\end{array}$} \\
\hline & $\begin{array}{l}\text { Indice } \\
\text { preconizado } \\
\text { em \% }\end{array}$ & $\begin{array}{l}\text { Valor } \\
\text { Absoluto }\end{array}$ & $\begin{array}{c}\text { Indice } \\
\text { preconizado } \\
\text { em } \%\end{array}$ & $\begin{array}{c}\text { Valor } \\
\text { Absoluto }\end{array}$ & $\begin{array}{l}\text { Indice } \\
\text { preconizado } \\
\text { em } \%\end{array}$ & $\begin{array}{l}\text { Valor } \\
\text { Absoluto }\end{array}$ \\
\hline $\begin{array}{l}\text { Enfermeira } \\
\text { Técnico de Enfermagem } \\
\text { Auxiliar de Enfermagem } \\
\text { Atendente de Enfermagem }\end{array}$ & $\begin{array}{r}5 \\
10 \\
25 \\
60\end{array}$ & $\begin{array}{r}1 \\
2 \\
5 \\
12\end{array}$ & $\begin{array}{l}\frac{15}{45} \\
40\end{array}$ & $\begin{array}{l}4 \\
\frac{4}{13} \\
11\end{array}$ & $\begin{array}{l}23 \\
3,5 \\
23,5 \\
50\end{array}$ & $\begin{array}{r}8 \\
1 \\
8 \\
17\end{array}$ \\
\hline TOT A L & 100,0 & 20 & 100,0 & 28 & 100,0 & 34 \\
\hline
\end{tabular}

\section{DISCUSSÃO}

No presente estudo de cálculo de pessoal realizado no Hospital Universitário por enfermeiras do Departamento de Assistência de Enfermagem, os resultados apresentados evidenciam uma variação entre os índices dos autores citådos e os dados reais do $\mathrm{HU}$.

O emprego de um determinado critério pode ser um ponto de partida para se efetuar estudo desta natureza, entretanto, as diferenças encontradas precisam ser analisadas à luz das características do local onde se realizou a pesquisa, isto porque existem fatores que influenciam sobremaneira o comportamento dos índices. Portanto, o emprego de um critério definido pode ser um ponto de partida para se calcular o pessoal para o serviço de enfermagem. Entretanto, a diferença do número de funcionários da $\mathrm{Clínica} \mathrm{Pe}$ diátrica, apresentada nos resultados: 29 para ABBOUD (s.d.) e 74 para o HU, deve ser vista através dos índices utilizados. Esta variação aconteceu não sర pela situaçáo real do nível de assistência percen- tual de leitos ocupados: $91,42 \%$, contra $80 \%$ preconizados pelos dois autores; $33 \%$ de absenteísmo encontrados no HU contra $20 \%$ referidos por $\mathrm{AB}$ BOUD (s.d.) e $30 \%$ por ALCALA (1982), mas principalmente pela classificação do tipo de cuidado. Comparando os resultados da Clínica Pediátrica com os da Clínica Médica, onde a diferença é menor, embora 0 índice de ocupação de leitos tenha se mantido em 91,42 e o absenteísmo foi maior (37\%), a influência da classificação por tipo de cuidado torna-se evidente. A partir destes achados, acredita-se que para estabelecer índices compatíveis com a realidade seja necessário manter uma classificação diária dos pacientes por tipo de cuidados e sugere-se, pelo menos, seis meses para que, de fato, este índice seja significativo. Isto porque podem ocorrer variaçōes quanto à gravidade dos pacientes, modificando os percentuais do tipo de cuidado.

Considera-se importante que a erfermeira que procede a classificação dos pacientes, obedeça a um padrão fixado para realizar este procedimento com objetividade e para diminuir a possibilidade de im- 
pressões subjetivas. É recomendável que de início o trabalho seja feito em dupla, até que se obtenha maior segurança.

Quanto ao índice de absenteísmo encontrado neste estudo, pode-se inferir, que sua superioridade em relação aos dados dos autores consultados, é decorrente do fato de ser o HU um hospital escola possuindo um quadro de funcionários predominantemente feminino.

Com relação aos índices de distribuição por categoria funcional, Tabelas 1 e 2, observa-se que ABBOUD (s.d.) e ALCALA (1982) estabeleceram percentuais menores para enfermeira do que os encontrados no HU, enquanto que neste as outras categorias têm percentuais inferiores a dos autores citados, o que pode minimizar os custos. Discorrer sobre esta diferença implicaria em questionar diretamente a qualidade de assistência.

A presença da enfermeira se faz necessária desde o treinamento de pessoal recém-admitido até o controle da qualidade de assistência prestada pelo serviço, incluindo a manutenção de um sistema de educação continuada, prestação de assistência seletiva e prescrição de enfermagem. Tarefas estas que não podem ser delegadas aos outros elementos da equipe e demandam não só um preparo profissional básico mas aperf eiçoamento contínuo do corpo de enfermeiras que respondem pela Instituição.

\section{CONCLUSĀO}

$\mathrm{O}$ número total de funcionários necessários à prestação de assistência de enfermagem em Clínica Pediátrica, segundo ABBOUD (s.d.) 29, ALCALA (1982) - 34, Departamento de Enfermagem do HU-74. Para a Clínica Médica foi de ABBOUD (s.d.) - 20, ALCALA (1982) - 28 e Departamento de Assistência a Enfermagem HU-34.
Existe acentuada diferença nos resultados obtidos com a utilização de diferentes índices para a Clínica Pediátrica. O mesmo não ocorreu na Clínica Médica, embora se evidencie razoável diferença.

A classificação dos pacientes da unidade ou instituição, por tipo de cuidado, torna o cálculo de pessoal mais proximo da necessidade real. Para tanto é necessário estabelecer índices que sejam compatŕveis com a realidade da Instituição.

\section{REFERÊNCIAS BIBLIOGRÁFICAS}

1 ABBOUD, M. Dotação de pessoal na organização hospitalar. São Paulo. Esc. Adm. de Emp. de São Paulo da FGV/HC da FMSUP, s. d. 9p. (apostila).

2 ALCALA, M.U. et alii. Cálculo de pessoal: estudo preliminar para estabelecimento de quadro de pessoal de Enfermagem na superintendência médico hospitalar de urgência. São Paulo, Superintendência Médico Hospitalar de Urgência, 1982. 47p.

3 ATALA, A. Cálculo de pessoal para os serviços paramédicos de um hospital geral de 300 leitos. Revista Paulista de Hospitais. 26(6): 244-56, jun. 1978.

4 DUTRA, V. de O. Administração de recursos no hospital. In: GONÇALVES, E.L. $O$ hospital e a visão administrativa contemporânea. São Paulo, Pioneira, 1983. cap. 2, p. 67-114 .

5 FELDMANN, M.A. Administração do senviço de Enfermagem. São Paulo, União Social Camiliana, s.d., 138p.

6 MEZOMO, J.C. Absenteísmo. In: tração dos recursos humanos no hospital. São Paulo, Centro São Camilo de Desenvolvimento em Administração da Saúde, 1981 p. 167

7 OGUISSO, T.; SCHMIDT, M.J. BOCHI, O.A. de C. O sistema empresarial da locação de pessoal de enf ermagem para o INPS. Revista Brasileira de Enfermagem, São Paulo, 23 $(1,2) ; 117-30$, jan./jun. 1970. 